PROCEEDINGS OF THE

AMERICAN MATHEMATICAL SOCIETY

Volume 130, Number 10, Pages 3117-3123

S 0002-9939(02)06438-9

Article electronically published on March 14, 2002

\title{
ANY 3-MANIFOLD 1-DOMINATES AT MOST FINITELY MANY 3-MANIFOLDS OF $S^{3}$-GEOMETRY
}

\author{
CLAUDE HAYAT-LEGRAND, SHICHENG WANG, AND HEINER ZIESCHANG \\ (Communicated by Ronald A. Fintushel)
}

\begin{abstract}
Any 3-manifold 1-dominates at most finitely many 3-manifolds supporting $S^{3}$ geometry.
\end{abstract}

\section{§1. INTRODUCTION}

A fundamental topic in topology is to determine whether there exists a map of nonzero degree between given closed orientable manifolds of the same dimension. A natural question in the topic is that for given manifold $M$, are there at most finite $N$ such that there is a degree one map $M \rightarrow N$ ?

For dimension 2, the answer is YES, since there is a map $f: F \rightarrow G$ of degree one between closed orientable surfaces if and only if the genus of $G$ is at most of the genus of $F$ and there are only finitely many surfaces with the bounded genus. For dimension at least 4 , it is still hopeless to get some general answer. For dimension 3 it becomes especially interesting after Thurston's revolutionary work, and has been listed as the 100th problem of 3-manifold theory in the Kirby's list.

Question 1 (3, 3.100, (Y. Rong)]). Let $M$ be a closed orientable 3-manifold. Are there only finitely many orientable closed 3-manifolds $N$ such that there exists a degree one map $M \rightarrow N$ ?

A closed orientable 3-manifold is called geometric if it admits one of the following geometries: $H^{3}, \widetilde{P L S_{2} R}, H^{2} \times E^{1}$, Sol, Nil, $E^{3}, S^{2} \times E^{1}, S^{3}$. Thurston's geometrization conjecture claims that any closed orientable 3 -manifold is either geometric or can be decomposed by the Kneser-Milnor sphere decomposition and the Jaco-Shalan-Johanson torus decomposition so that each piece is geometric. (For details see [11.)

All geometric 3-manifolds with finite fundamental group are exactly those with $S^{3}$-geometry. All geometric 3-manifolds are Seifert manifolds except those that carry hyperbolic geometry or Sol geometry.

Many partial answers to Question 1 have been obtained: Any sequence $M_{1} \rightarrow$ $M_{2} \rightarrow M_{3} \rightarrow \ldots$ of degree one maps between Haken 3-manifolds must be stable when $n$ is large $([8],[14])$. The answer to Question 1 is affirmative if both, the

Received by the editors November 17, 2000 and, in revised form, May 23, 2001.

2000 Mathematics Subject Classification. Primary 55M25, 54C05, 57M05.

Key words and phrases. 3-manifold, degree one map.

The second author was partially supported by MSTC and Outstanding Youth Fellowships of NSFC.

(C)2002 American Mathematical Society 
domain and the target, are Seifert manifolds with infinite fundamental group ([9], [10]) or the domain is non-Haken and the target is hyperbolic [7].

All partial results above need some conditions posed on the domain. A more recent result is that Question 1 has a positive answer when the targets are either hyperbolic [13, Sol manifolds, or Seifert manifolds with infinite fundamental group [16], where no conditions are posed on the domain. A main result of this article is that Question 1 has a positive answer when the targets support $S^{3}$ geometry.

For simplicity, we use the following terminology introduced in [1]. For an integer $d>0$, a closed orientable 3-manifold $M d$-dominates a closed orientable 3-manifold $N$ if there is a continuous map $f: M \rightarrow N$ of degree $d$. We also assume that all integers $d, n, q$ involved in the results of this paper are positive. The modification for the negative case can be made easily by reversing the orientations.

Theorem 1. An orientable closed 3-manifold M 1-dominates at most finitely many 3-manifolds supporting $S^{3}$-geomerty.

Combining Theorem 1 with the results of [13] and [16], we know that Question 1 has a positive answer when the targets are geometric 3-manifolds.

To prove Theorem 1 we need the following result, which is independently interesting.

Theorem 2. A closed orientable 3-manifold $M d$-dominates the lens space $L(n, q)$ if and only if there is an element $\alpha \in$ Tor $H_{1}(M, \mathbb{Z})$ such that $\alpha \odot \alpha=\frac{d q}{n}$ in $\mathbb{Q} / \mathbb{Z}$, where $\alpha \odot \alpha$ is the self-linking number of $\alpha$.

Theorem 2 is an extension of the main result in 4. To prove Theorem 2, some careful arguments for the orders of (co-)homology classes are needed. For this purpose, we present a more direct and geometric proof than the one given in [4].

The paper is organized as follows. Theorem 2 is proved in Section 2 and Theorem 1 is proved in Section 3. The necessary preparations are also given in each section, and therefore the paper is almost self-contained. We assume that all objects below are oriented with compatible orientations.

\section{§2. Proof of Theorem 2}

1. Lens spaces $L(n, q)$ and $L^{\infty}(n)=K\left(Z_{n}, 1\right)$. Let $S^{2 l+1} \subset \mathbb{C}^{l+1}$ be the unit sphere in $(l+1)$ complex space. Then $\tau: C^{l+1} \rightarrow C^{l+1}$ given by

$$
\tau\left(z_{1}, z_{2}, \ldots, z_{l+1}\right)=\left(e^{2 \pi q / n} z_{1}, e^{2 \pi / n} z_{2}, \ldots, e^{2 \pi / n} z_{l+1}\right)
$$

defines a free action on $S^{2 l+1}$. Denote the quotient $S^{2 l+1} / \tau$ by $L^{2 l+1}(n, q)$, and the classical lens space $L^{3}(n, q)$ is still denoted as $L(n, q)$.

Let $L^{\infty}(n)=\bigcup_{l=1}^{\infty} L^{2 l+1}(n, q)$ be a $K\left(Z_{n}, 1\right)$ space, that is, $\pi_{1}=Z_{n}$ and the higher homotopy groups are vanished. Note that $L(n, q)$ is the 3-skeleton of $L^{\infty}(n)$.

An alternative description of $L(n, q)$ is identifying the boundary of two solid tori $V_{1}$ and $V_{2}$ such that the meridian of $V_{1}$ is matched with $(q, n)$ curve in $\partial V_{2}$, where on the boundary $\partial V_{2}$ a meridian-longitude system is chosen.

2. The linking pair of 3-manifolds. Suppose $M$ is a closed oriented 3-manifold. Suppose $\alpha \in \operatorname{Tor} H_{1}(M, Z)$ is an element of order dividing $n$. Let $a$ be a 1-cycle representing $\alpha$, then na bounds a 2-chain $A$. Push $a$ slightly so that $A$ and $a$ are in general position. Then the self-linking number of $\alpha$, denoted as $\alpha \odot \alpha$, is defined by $\frac{1}{n} A \cdot a$, where "." indicates the algebraic intersection number. (See [12] Section $77]$. 
3. The Bockstein homomorphism. For the chain complex $C_{*}=C_{*}(M)$, the short exact sequence

$$
0 \rightarrow \mathbb{Z} \otimes C_{*} \rightarrow \mathbb{Z} \otimes C_{*} \rightarrow \mathbb{Z}_{n} \otimes C_{*} \rightarrow 0
$$

provides the long exact homology sequence and cohomology sequence, and the $\partial$ operators are the Bockstein homomorphisms $\beta^{*}: H^{1}\left(M, \mathbb{Z}_{n}\right) \rightarrow H^{2}(M, \mathbb{Z})$ and $\beta_{*}: H_{2}\left(M, \mathbb{Z}_{n}\right) \rightarrow H_{1}(M, \mathbb{Z})$ We have

$$
\beta^{*} \circ D=D \circ \beta_{*}
$$

up to sign, where $D$ is the isomorphism of the Poincaré duality [5] Lemma 69.2].

4. Relationship between linking pair and Bockstein homomorphism. A geometric feature of $\beta_{*}$ is that (check [5. Step 1, p. 137])

$$
\beta_{*}(c)=[\partial C / n]
$$

for any $c \in H_{2}\left(M, \mathbb{Z}_{n}\right)$, where $\partial$ is the boundary operator on 2-chain complex $C_{2}(M)$ and $C \in C_{2}(M)$ is a $\bmod n$ cycle representing $c$. Clearly the order of $\beta_{*} c \in \operatorname{Tor} H_{1}(M, \mathbb{Z})$ divides $n$. Moreover, we have

$$
\frac{1}{n} c \cdot \beta_{*}(c)=\beta_{*}(c) \odot \beta_{*}(c)
$$

and

$$
D^{-1}(c) \cup \beta^{*} \circ D^{-1}(c)=c \cdot \beta_{*}(c)[M],
$$

where $[M] \in H^{3}\left(M, \mathbb{Z}_{n}\right)$ is the fundamental class. Equality (3) follows from (2) and equality (4) follows diagram (1).

Lemma 0. If there is $\alpha \in \operatorname{Tor} H_{1}(M, \mathbb{Z})$ such that $\alpha \odot \alpha=\frac{d q}{n}$, where $q$ and $n$ are coprime, then the order of $\alpha$ divides $n$ and is divided by $\frac{n}{\operatorname{gcd}(n, d)}$.

Proof. The order of $\alpha$ dividing $n$ follows from the definition of the linking pair, and the order of $\alpha$ is divided by $\frac{n}{\operatorname{gcd}(n, d)}$ follows from that the order of $\alpha \odot \alpha$ is $\frac{n}{\operatorname{gcd}(n, d)}$ in $\mathbb{Q} / \mathbb{Z}$ and the order of $\alpha \odot \alpha$ divides the order of $\alpha$.

Proof of Theorem 2. Suppose that there is a map $f: M \rightarrow L(n, q)$ of degree $d$.

Recall $L(n, q)$ is obtained by identifying two solid tori $V_{1}$ and $V_{2}$ such that the meridian of $V_{1}$ is matched with the $(q, n)$ curve in $\partial V_{2}$, where in the boundary $V_{2}$ a meridian-longitude system is chosen. Let $Y$ be the centerline of $V_{2}$. Then note first $n Y$ and $(q, n)$-curve are homological in $V_{2}$ and therefore they bound a 2-complex $B^{\prime}$. Since the $(q, n)$-curve on $\partial V_{2}$ bounds the meridian disc $B$ of $V_{1}, n Y$ bounds a 2-complex $C=B^{\prime}+B$, which is a $\bmod n$ cycle.

Let $\gamma=[Y]$ in $H_{1}(L(n, q), \mathbb{Z})$, and $c=[C] \in H_{2}\left(L(n, q), \mathbb{Z}_{n}\right)$. Clearly $\gamma$ is of order $n$, and $\beta_{*}(c)=\gamma$ by (2). By direct geometric observation we have $C \cdot Y=q$, and therefore $\gamma \odot \gamma=\frac{q}{n}$ by (3). Let $y=D^{-1}(c) \in H^{1}\left(L(n, q), \mathbb{Z}_{n}\right)$. Then $y$ has order $n$ and, by (4), $y \cup \beta^{*}(y)=q[L(n, q)]$. Let $x=f^{*}(y) \in H^{1}\left(M, \mathbb{Z}_{n}\right)$. Then the order of $x$ divides $n$. Since $f$ is of degree $d$ we have

$$
x \cup \beta^{*}(x)=f^{*}(y) \cup \beta^{*}\left(f^{*}(y)\right)=f^{*}\left(y \cup \beta^{*}(y)\right)=f^{*}(q[L(n, q)])=d q[M] .
$$

Let $\alpha=\beta_{*} \circ D(x)$. Then $\alpha$ has order dividing $n$. By (3), (1), (5) and (4), we have

$$
\alpha \odot \alpha=\frac{1}{n}\left(\beta_{*} \circ D(x)\right) \cdot D(x)=\frac{d q}{n} .
$$


Next we prove the other direction. If $\alpha \in H_{1}(M, \mathbb{Z})$ has the properties that $\alpha \odot \alpha=\frac{d q}{n}$, then the order of $\alpha$ divides $n$ (Lemma 0) and therefore there is $[c] \in H_{2}\left(M, \mathbb{Z}_{n}\right)$ such that $\beta_{*}([c])=\alpha$. Let $x=D^{-1}(c) \in H^{1}\left(M, \mathbb{Z}_{n}\right)$. Then similarly, we have $x \cup \beta^{*}(x)=d q[M]$.

We consider the Eilenberg-MacLane space $K\left(\mathbb{Z}_{n}, 1\right)=L^{\infty}(n)$. Let $j: L(n, q) \rightarrow$ $K\left(\mathbb{Z}_{n}, 1\right)$ be the inclusion. Then

$$
j^{*}: H^{1}\left(K\left(\mathbb{Z}_{n}, 1\right), \mathbb{Z}_{n}\right) \rightarrow H^{1}\left(L(n, q), \mathbb{Z}_{n}\right)
$$

is an isomorphism. Let $y$ be a generator of $H^{1}\left(L(n, q), \mathbb{Z}_{n}\right)$ such that $y \cup \beta^{*}(y)=$ $q[L(n, q)]$, and choose $z=j^{*-1}(y) \in H^{1}\left(K\left(\mathbb{Z}_{n}, 1\right), \mathbb{Z}_{n}\right)$ as a generator. By [15] Theorem 10 (p. 428)], $x$ determines a map $g_{x}: M \rightarrow K\left(\mathbb{Z}_{n}, 1\right)$, uniquely up to homotopy, such that $g_{x}^{*}(z)=x$. Since $M$ is of dimension $3, g_{x}$ can be deformed such that its image lies in $L(n, q)$, the 3 -skeleton of $K\left(\mathbb{Z}_{n}, 1\right)$. Thus there is a map $g: M \rightarrow L(n, q)$ such that $j \circ g=g_{x}$. Hence, in cohomology level we have

$$
g^{*}(y)=g^{*} j^{*}(z)=g_{x}^{*}(z)
$$

and

$$
\operatorname{deg}(g) q[M]=g^{*}(q[L(n, q)])=g^{*}\left(y \cup \beta^{*}(y)\right)=x \cup \beta^{*}(x)=d q[M] .
$$

Since $\operatorname{gcd}(n, q)=1$, we have $\operatorname{deg}(g) \equiv d \bmod n$, i.e., $\operatorname{deg}(g)=d+\ln$ for some integer $l$. Starting with $g$ and a map $h: S^{3} \rightarrow L(n, q)$ of degree $-n l$, we can now construct a map $f=g \# h: M \# S^{3} \rightarrow L(n, q)$ of degree $d$.

Corollary 1. Any closed orientable 3-manifold $M$ d-dominates at most finitely many lens spaces.

Proof. $\operatorname{Tor} H_{1}(M, \mathbb{Z})$ is finite and therefore there are only finitely many integers $l$ dividing the order of $\operatorname{Tor} H_{1}(M, \mathbb{Z})$. For each integer $l$ the number of integers $n$ such that $l=k n / \operatorname{gcd}(n, d)$ is finite since $\operatorname{gcd}(n, d) \leq d$. For each $n$, there exist only finitely many lens spaces $L(n, q)$. Corollary 1 now follows from Theorem 2 and Lemma 0.

\section{§3. Proof of Theorem 1}

1. Presentations and standard forms of Seifert manifolds. Each oriented Seifert manifold $M$ with base $F_{g}$, the closed orientable surfaces of genus $g$, has a presentation $M=\left(g ; a_{0}, b_{0} ; a_{1}, b_{1} ; \ldots ; a_{n}, b_{n}\right)$, which indicates the construction of $M$ as follows: Let $F$ be a $n+1$ punctured $F_{g}$ with boundary components $c_{0}, c_{2}, \ldots, c_{n}$. Then on $T_{i}$, the $i$ th torus boundary component of $F \times S^{1}$, the pair $\left(c_{i}, h_{i}\right)$ forms a coordinate system on $T_{i}$, where $h_{i}$ is a $S^{1}$-fiber on $T_{i}$. Then $M$ is obtained from $F \times S^{1}$ by identifying the boundary of a solid torus $S_{i}$ and $T_{i}$ such that the meridian of $S^{i}$ is matched with the $\left(a_{i}, b_{i}\right)$-curve, $a_{i}>0$, for each $i$. Clearly $\operatorname{gcd}\left(a_{i}, b_{i}\right)=1$. Since $a_{i}>0$, the circle fibration on $F \times S^{1}$ can be extended to each $S_{i}$, where the centerline of $S^{i}$ is a regular fiber if $a_{i}=1$, and a singular fiber if $a_{i}>1$.

The presentations of a Seifert manifold $M$ is not unique. However, the following fact is known:

Lemma 1 ([6]). Suppose $M=\left(g ; a_{0}, b_{0} ; a_{1}, b_{1} ; \ldots ; a_{n}, b_{n}\right), M^{\prime}=\left(g^{\prime} ; a_{0}^{\prime}, b_{0}^{\prime} ; a_{1}^{\prime}, b_{1}^{\prime}\right.$; $\left.\ldots ; a_{m}^{\prime}, b_{m}^{\prime}\right)$ are oriented Seifert manifolds with oriented base, $n \leq m$. Then $M$ and $M^{\prime}$ are orientation preserving homeomorphic by a fiber-preserving homeomorphism 
if and only if the following conditions hold after adding some pair " $(1,0)$ "s to $M=$ $\left(g ; a_{0}, b_{0} ; a_{1}, b_{1} ; \ldots ; a_{n}, b_{n}\right)$ and re-indexing those pairs if necessary.

1. $g=g^{\prime}$,

2. $m=n$,

3. $a_{i}=a_{i}^{\prime}, b_{i}=b_{i}^{\prime}+k_{i} a_{i}$ for all $i$ and $\sum_{i=1}^{n} k_{i}=0$.

Recall that a presentation of the Seifert manifold is in standard form, if $a_{0}=1$, $a_{i}>b_{i}>0$. A given Seifert manifold $M$ has unique standard form. The standard form is often written as $\left(g ; 1, l ; a_{1}, b_{1} ; \ldots ; a_{n}, b_{n}\right)$.

2. The order of the homology of Seifert manifolds supporting $S^{3}$-geometry. If $M$ admits $S^{3}$-geometry, then $M$ is either a lens space or $M$ has the standard form $\left(0 ; 1, l ; a_{1}, b_{1} ; a_{2}, b_{2} ; a_{3}, b_{3}\right)$, a Seifert fibered space with base $S^{2}$ and 3 singular fibers, where $\left(a_{1}, a_{2}, a_{3}\right)$ is one of the following: $(2,2, n),(2,3,3),(2,3,4),(2,3,5)$.

If $M=\left(0 ; 1, l ; a_{1}, b_{1} ; a_{2}, b_{2} ; a_{3}, b_{3}\right)$, then

$$
\left.\pi_{1}(M)=\left\langle x_{1}, x_{2}, x_{3}, h\right|\left[x_{j}, h\right], x_{j}^{a_{j}} h^{b_{j}} \text { for } 0 \leq j \leq 3, x_{1} x_{2} x_{3} h^{-l}\right\rangle,
$$

By abelianizing $\pi_{1}(M)$, one can calculate that the order of $H_{1}(M, \mathbb{Z})$ is

$$
\left|a_{1} a_{2} a_{3}\left(\frac{b_{1}}{a_{1}}+\frac{b_{2}}{a_{2}}+\frac{b_{3}}{a_{3}}+l\right)\right| .
$$

An easy consequence of this fact is

Lemma 2. For any given positive integers $C, a_{1}, a_{2}$ and $a_{3}$, there are only finitely many Seifert manifolds $M$ with standard form $\left(0 ; 1, l ; a_{1}, b_{1} ; a_{2}, b_{2} ; a_{3}, b_{3}\right)$ such that the order of $H_{1}(M, \mathbb{Z})$ is smaller than $C$.

We also need the following known fact (see [2, I.2.5], for example).

Lemma 3. Let $M$ and $N$ be orientable closed 3-manifolds. If $f: M \rightarrow N$ is a degree one map, then $\operatorname{Tor} H_{1}(M, \mathbb{Z})=A \oplus \operatorname{Tor} H_{1}(N, \mathbb{Z})$.

3. $Z_{2}$ action on Seifert manifolds from the fiber direction. Suppose $M=$ $F \times S^{1} \cup\left(\bigcup_{0}^{n} S_{i}\right)=\left(g ; a_{0}, b_{0} ; a_{1}, b_{1} ; \ldots ; a_{n}, b_{n}\right)$, where $F$ is the $(n+1)$-punctured $F_{g}$. Consider the $\mathbb{Z}_{2}$ action $\tau^{\prime}$ on $F \times S^{1}$ which induces the identity on $F$ and rotation on each $S^{1}$ fiber by $\pi$.

Lemma 4. The above $\mathbb{Z}_{2}$ action can extend to a $Z_{2}$ action $\tau$ on $M=\left(g ; a_{0}, b_{0}\right.$; $\left.a_{1}, b_{1} ; \ldots ; a_{n}, b_{n}\right)$. Moreover, the quotient space $M / \tau$ is a Seifert manifold with representation $\left(g ; a_{0}^{\prime}, b_{0}^{\prime} ; \ldots ; a_{n}^{\prime}, b_{n}^{\prime}\right)$, where $a_{i}^{\prime}=\frac{a_{i}}{2}, b_{i}^{\prime}=b_{i}$ if $a_{i}$ is even and $a_{i}^{\prime}=a_{i}$, $b_{i}^{\prime}=2 b_{i}$ if $a_{i}$ is odd.

Proof. The $\mathbb{Z}_{2}$-action $\tau^{\prime}$ on $T_{i}$, the $i$ th torus boundary component, is free and orientation preserving. Hence it provides a free and orientation preserving $\mathbb{Z}_{2}$ action on $\partial S_{i}$ via the identification map. The latter can extend to a $Z_{2}$-action $\tau_{i}$ on $S_{i}$. Therefore $\tau^{\prime}$ and $\tau_{i}$ together give an $\mathbb{Z}_{2}$ action on $M$ which extends $\tau^{\prime}$. And we have

$$
M / \tau=\left(F \times S^{1} / \tau^{\prime}\right) \cup\left(\bigcup_{i=0}^{n} S_{i} / \tau_{i}\right)
$$

Note that (1) $S_{i}^{\prime}=S_{i} / \tau_{i}$ is still a solid torus and the quotient map $S_{i}$ sends the meridian disc to the meridian disc; (2) $F \times S^{1} / \tau^{\prime}=F \times S^{1}$ and the quotient map $T_{i} \rightarrow T_{i} / \tau^{\prime}=T_{i}^{\prime}$ for each boundary torus sends the $\left(a_{i}, b_{i}\right)$ curve on $T$ to the 
$\left(\frac{a_{i}}{2}, b_{i}\right)$ curve on $T^{\prime}$ if $a_{i}$ is even and to the $\left(a_{i}, 2 b_{i}\right)$ curve if $a_{i}$ is odd. Then the lemma follows.

Proof of Theorem 1. Suppose $M$ 1-dominates infinitely many Seifert manifolds $N_{j}$ with finite fundamental group. These targets form the following two classes:

Class (1). $N_{j}$ is a lens space $L(n, q)$ with $\left|H_{1}\left(N_{j}, \mathbb{Z}\right)\right|=n$.

Class (2). One of the spaces with standard forms $\left(0 ; 1, l ; 2,1 ; 3, b_{2} ; 3, b_{3}\right)$ with $\left|H_{1}\left(N_{j}, \mathbb{Z}\right)\right|=\left|9+6 b_{2}+6 b_{3}+18 l\right|$, or $\left(0 ; 1, l ; 2,1 ; 3, b_{2} ; 4, b_{3}\right)$ with $\left|H_{1}\left(N_{j}, \mathbb{Z}\right)\right|=\mid 12+$ $8 b_{2}+6 b_{3}+24 l \mid$, or $\left(0 ; 1, l ; 2,1 ; 3, b_{2} ; 5, b_{3}\right)$ with $\left|H_{1}\left(N_{j}, \mathbb{Z}\right)\right|=\left|15+10 b_{2}+6 b_{3}+30 l\right|$.

Class (3). $N_{j}$ is a prism space $\left(0 ; 1, l ; 2,1 ; 2,1 ; n, b_{3}\right)$ with $\left|H_{1}\left(N_{j}, \mathbb{Z}\right)\right|=4|n+k|$, where $k=b_{3}+n l$.

By Lemma $3,\left|H_{1}\left(N_{j}, \mathbb{Z}\right)\right|$ is bounded by $\left|\operatorname{Tor} H_{1}(M, \mathbb{Z})\right|$. By Lemma 2 , there are at most finitely many $N_{j}$ in classes (1) and (2).

We now handle the manifolds in class (3). The important difference to cases (1) and $(2)$ is that there are infinitely many distinct prism spaces with the same first homology group because the conditions are only that $n>0$ and $0<b_{3}<n$ while $l$, hence $k=b_{3}+n l$, is arbitrary.

For convenience we represent the manifolds in class (3) by $(0 ; 1,0 ; 2,1 ; 2,1 ; n, k)$ with first homology of order $4|k+n|$; here $k$ can be any integer prime to $n$ and $l$ vanishes.

Assume that $M$ 1-dominates infinitely many distinct prism manifolds. By Lemma $3,\left|n_{j}+k_{j}\right|$ is bounded by the order of $H_{1}(M, \mathbb{Z})$. By passing to a subsequence, we may assume that $n_{j}+k_{j}=C$ is fixed. Moreover, we may assume that all $n_{j}$ are different.

Consider the $\mathbb{Z}_{2}$ action on $\left(0 ; 1,0 ; 2,1 ; 2,1 ; n_{j}, k_{j}\right)$ which induces the identity on the base orbifold and rotates on each regular $S^{1}$ fiber by $\pi$. Then the quotient space $\left(0 ; 1,0 ; 2,1 ; 2,1 ; n_{j}, k_{j}\right) / \mathbb{Z}_{2}$ has presentation $\left(0 ; 1,0 ; 1,1 ; 1,1 ; n_{j}, 2 k_{j}\right)$ if $n_{j}$ is odd and $\left(0 ; 1,0 ; 1,1 ; 1,1 ; n_{j} / 2, k_{j}\right)$ if $n_{j}$ is even by Lemma 4 . Now

$$
\left(0 ; 1,0 ; 1,1 ; 1,1 ; n_{j}, 2 k_{j}\right)=\left(0 ; 1,2 ; n_{j}, 2 k_{j}\right)=L\left(2\left(k_{j}-n_{j}\right), *\right)
$$

where $* \in \mathbb{Z}$. The first equality follows from Lemma 1 , and the second follows by counting the intersection numbers of two meridians and

$$
\left(0 ; 1,0 ; 1,1 ; 1,1 ; n_{j} / 2, k_{j}\right)=\left(0 ; 1,2 ; n_{j} / 2, k_{j}\right)=L\left(k_{j}-n_{j}, *\right) .
$$

This construction provides maps of $M$ of degree 2 onto the above lens spaces. If infinitely many of these $n_{j}$ are odd, then $M$ 2-dominates the family of lens spaces $\left\{L\left(2\left(k_{j}-n_{j}\right), *\right)\right\}$. By Theorem $2,2\left|k_{j}-n_{j}\right|$ must be bounded. By passing to a subsequence we may therefore assume that $2\left(k_{j}-n_{j}\right)=C^{\prime}$ for some constant $C^{\prime}$. Now substitute $2 n_{j}=2 k_{j}-C^{\prime}$ into $n_{j}+k_{j}=C$ to deduce that $k_{j}$ and, hence, $n_{j}$ are constant, which is a contradiction.

If infinitely many of these $n_{j}$ are even, then $M$ 2-dominates the family $\left\{L\left(k_{j}-\right.\right.$ $\left.\left.n_{j}, *\right)\right\}$ of lens spaces. Now one obtains a contradiction as above by again showing that the $n_{j}$ coincide for infinitely many $j$.

\section{REFERENCES}

[1] Boileau M. and Wang S.C. Nonzero degree maps and surface bundles over $S^{1}$, J. Diff. Geom. 43 (1996), 789-908. MR 98g:57023

[2] Browder, W.: Surgery on simply-connected manifolds. Berlin-Heidelberg-New York: Springer 1972. MR 50:11272 
[3] Kirby, R.: Problems in low-dimensional topology. Geometric topology, Edited by H. Kazez, AMS/IP Vol. 2., International Press, 1997. MR 98f:57001

[4] Hayat-Legrand, C.; Wang, S.C. and Zieschang, H.: Degree one maps onto Lens Spaces. Pacific J. Math. 176, 19-32 (1996) MR 98b:57030

[5] Munkres, J., Elements of Algebraic Topology. Addison-Wesley Publish Company 1984. MR 85m:55001

[6] Neumann, W.D. and Raymond, F., Seifert manifolds, plumbing, $\mu$-invariant and orientation reversing maps, Lect. Note in Math. 664, 162-195, Springer, 1978. MR 80e:57008

[7] Reid, A. and Wang, S.C., Non-Haken 3-manifolds is not larger with respect to mappings of nonzero degree, Comm. Analy. Geom. Vol. 7, No. 1, 105-132 (1999). MR 2000c:57042

[8] Rong, Y. Degree one maps between geometric 3-manifolds. Trans. Amer. Math Soc, 322 411-436 (1992). MR 92j:57007

[9] Rong, Y. Maps between Seifert fibered spaces of infinite $\pi_{1}$. Pacific J. Math. 160, 143-154 (1993). MR 94e:55026

[10] Rong, Y. Degree one maps of Seifert manifolds and a note on Seifert volume, Topology and its Application. Vol. 64 No. 2 191-200 (1995). MR 96c:57034

[11] Scott, G.P., The geometries of 3-manifolds. Bull. London Math. Soc. 15, 401-487 (1983).

[12] Seifert, H. and Threlfall, W., A text book of topology (English transl.) Academic Press 1980. MR 82b:55001

[13] Soma, T., Nonzero degree maps to hyperbolic 3-manifolds, J. Diff. Geom. 49 (1998), 517-546. MR 2000b:57034

[14] Soma, T., Sequences of degree-one maps between geometric 3-manifolds, Math. Annalen. 316, (2000) 733-742. MR 2001b:57039

[15] Spanier, E., Algebraic Topology. McGraw-Hill Book Comp., New York, N.Y. 1966. MR 35:1007

[16] Wang, S.C. and Zhou, Q., Any 3-manifold 1-dominates only finitely Seifert manifolds with infinite $\pi_{1}$. Math. Annalen, in press.

Department of Mathematics, University of Sabatier, Toulouse 31062, France

E-mail address: hayat@picard.ups-tlse.fr

Department of Mathematics, Peking University, Beijing 100871, People's Republic of CHINA

E-mail address: wangsc@math.pku.edu.cn

Department of Mathematics, Ruhr University, Bochum 44780, Germany

E-mail address: marlene.schwarz@rz.ruhr-uni-bochum.de 